\title{
Performance Analysis of Cellular Radio Access Networks Relying on Control- and User-Plane Separation
}

\author{
Kai Liang, Member, IEEE, Gexian Liu, Liqiang Zhao, Member, IEEE, Xiaoli Chu, Member, IEEE, Shuai Wang, \\ Lajos Hanzo, Fellow, IEEE
}

\begin{abstract}
In a cellular radio access network (RAN) relying on control- and user-plane separation, control base stations (CBSs) form the control plane (CP) and take care of control coverage as well as low speed data delivery. In contrast, traffic base stations (TBSs) in the user plane (UP) are used for transmitting high speed data. Based on queuing theory and stochastic geometry, we derive the coverage probability of the $\mathrm{CP}$ under non-line-ofsight $(\mathrm{NLOS})$ transmissions, and derive the energy efficiency of the UP under coexisting line-of-sight $(\operatorname{LoS})$ and NLoS propagation modes. Numerical results show that when the traffic load is high, the coverage probability of the $\mathrm{CP}$ increases with the increasing CBSs' data rate or the density of CBSs. We also found that the maximum energy efficiency of the UP can be achieved by optimizing the TBS density, while achieving a high area spectral efficiency.
\end{abstract}

Index Terms-Cellular RANs, control- and user-plane separation, coverage probability, energy efficiency, LoS, NLoS, queuing theory

\section{INTRODUCTION}

In response to the enormous increase in mobile data traffic, cellular radio access networks (RANs) relying on control- and user-plane separation (CUPS) have been introduced in [1]. CUPS decouples the control coverage from traffic coverage, by which the traffic coverage may be adapted in response to both the dynamics of mobile traffic characteristics and to the prevalent quality of service (QoS) requirements. A cellular RAN relying on CUPS consists of two kinds of base stations (BSs). Specifically, the control base stations (CBSs) of the control plane (CP) deliver control signals (such as cell-specific reference signals and radio resource control, see [2] for details) and low rate signals at a low carrier frequency, while traffic

Copyright (c) 2015 IEEE. Personal use of this material is permitted. However, permission to use this material for any other purposes must be obtained from the IEEE by sending a request to pubs-permissions@ieee.org.

This work was supported in part by National Natural Science Foundation of China (61771358), National Natural Science Foundation of Shaanxi Province (2018JM6052), the 111 Project (B08038), China Postdoctoral Science Foundation (2017M613074), and Fundamental Research Funds for the Central Universities (JB190104).

Kai Liang, Gexian Liu, Liqiang Zhao, and Shuai Wang are with the State Key Laboratory of Integrated Service Networks, Xidian University,Xian 710071, China (e-mail:kliang@xidian.edu.cn; gexianliu@yeah.net; lqzhao@mail.xidian.edu.cn; ws504881821@163.com). Xiaoli Chu is with department of Electronic and Electrical Engineering, University of Sheffield, UK (x.chu@sheffield.ac.uk). Lajos Hanzo is with the School of Electronics and Computer Science, University of Southampton, Southampton SO17 1BJ, U.K (lh@ecs.soton.ac.uk).

L. Hanzo is grateful for the support of the EPSRC projects EP/Noo4558/1, EP/PO34284/1 and of the ERC's Advanced Fellow Award QuantCom. base stations (TBSs) in the user plane (UP) provide high-rate data transmissions at a high carrier frequency.

The idea of physical separation the CP and the UP is closely in line with $5 \mathrm{G}$ standards in terms of Non-Standalone (NSA) (3GPP TR 38.801). In the NSA mode, the BSs are classified into two types that are in charge of control and information transmissions, respectively. CUPS achieves the advantages of reduced design complexity and expenditure of BS, simplified network management through software defined networking (SDN) [3] and reduced energy consumption at TBSs [1]. Moreover, the idea of CUPS will be used in B5G/6G as the key enabling technology of open network architecture [4], which facilitates the network with the characteristics of fast-development, flexibility, re-configurability and economy.

The system model of cellular RANs relying on CUPS studied in [5]-[7] assumes that the fading channel models of both planes are the same and that only non-line-of-sight (NLoS) transmissions exist. In [5] and [6], the authors investigated caching strategies of cache-enabled hyper-cellular networks (HCNs) based on the CUPS. Zhang et al. [7] studied the benefits of the sleep scheme of small cells in HCNs, where the densities of macro BS users and small cell users are proportional to the traffic load in each layer. The channel models used in aforementioned literatures only considered the NLoS path loss mode. However, the shorter transmission distance in the UP results in predominantly line-of-sight (LoS) transmissions [8]. In [9], the authors studied CUPS based RAN slicing, and the validity of CUPS was proved by a testbed facilitated by software defined radio and LTE Release 8 .

The main contributions of this treatise are summarized as follows. Firstly, upon invoking stochastic geometry, we provide an analytical model for cellular RANs relying on the beneficial feature of CUPS. Secondly, the rate requirements of users in the CP and the UP are defined independently with the aid of queuing theory. Thirdly, the CP and the UP have different channel models, where the UP experiences both LoS and NLoS transmissions, while the CP mainly exhibits NLoS propagation. Finally, since providing reliable coverage is of the highest priority, we analyze the coverage probability for the CP. By contrast, energy efficiency is studied for the UP analysis because of the high-rate data requirement. Our numerical results show that the coverage probability of the $\mathrm{CP}$ can be improved upon increasing the service rates or the density of the CBSs, and there exists a TBS density that maximizes the energy efficiency of the UP, while additionally 
improving the area spectral efficiency (ASE). To the best of our knowledge, this is the first contribution jointly modelling and analyzing cellular radio access networks (RANs) relying on CUPS, while considering different rate requirements in LoS/NLoS scenarios in the CP as well as in the UP and invoking stochastic geometry and queuing theory.

\section{System Model AND Channel Models}

We consider downlink (DL) transmissions in a cellular RAN relying on CUPS, where CBSs and TBSs are deployed following two independent homogeneous spatial Poisson point processes (PPPs), i.e., $\Psi_{C}$ and $\Psi_{T}$, on the plane $\mathbb{R}^{2}$ with density of $\lambda_{C}$ and $\lambda_{T}$, respectively. The cellular users are distributed following another independent PPP $\Psi_{U}$ with density $\lambda_{u}$. All users receive control signals from their serving CBSs. The users with low and high user data-rate requirements are connected to their serving CBSs and TBSs, respectively. We denote the probability of a user requiring a high DL data-rate by $p_{u}$. The time intervals of each user accessing its serving CBS and TBS obey exponential distributions with their mean value of $1 / \lambda_{a}(\mathrm{sec})$ and $1 / \lambda_{b}(\mathrm{sec})$, respectively. The arrival rates of a user's request at a CBS or a TBS are $\lambda_{a}$ and $\lambda_{b}$, respectively. When a user is allocated a channel from its serving CBS and TBS, its channel holding time follows two independent exponential distributions, which have a mean of $1 / \mu_{c}(\mathrm{sec})$ and $1 / \mu_{t}(\mathrm{sec})$, respectively.

\section{A. Traffic Model for the Control Plane}

According to the Erlang model[10], the total user traffic load over an area covered by a CBS is $\nu^{C}=\lambda_{a} N_{C}$, where $N_{C}=\lambda_{u} / \lambda_{C}$ is the average number of users served by each CBS. Based on the queuing theory, the queuing model of each CBS is $M / M / C_{C} / C_{C}$ associated with an Erlang load of $a_{c}=$ $\nu^{C} / \mu_{c}$, where we assumed that each CBS has $C_{C}$ channels.

The probability that $j$ channels are being used at a CBS is

$$
\phi_{j}^{C}=\frac{\left(a_{c}\right)^{j}}{j !} \phi_{0}^{C}=\frac{\left(a_{c}\right)^{j}}{j !}\left(\sum_{k=0}^{C_{C}} \frac{\left(a_{c}\right)^{k}}{k !}\right)^{-1}
$$

where $j \in\left\{1, \cdots, C_{C}\right\}$. We denote the probability of a neighboring CBS interfering with a typical CBS by $\rho^{C}$ [10], where $\rho^{C}=\sum_{j=1}^{C_{C}} \frac{j}{C_{C}} \phi_{j}^{C}$. Therefore, the interfering CBSs are distributed following a PPP, which is denoted by $\Psi_{I}^{C}$, with a density of $\lambda_{I}^{C}=\rho^{C} \lambda_{C}$. The CBSs DL transmission power on each channel is set to $P_{t}^{C}$.

\section{B. Channel Model for the Control Plane}

The DL channels of CBSs exhibit both small-scale Rayleigh fading. We assume NLoS transmissions with a path loss exponent of $\alpha$ in the $\mathrm{CP}$ due to the larger coverage area. Specifically, the path gain in the $\mathrm{CP}$ is given by

$$
\Theta^{C P}=A r^{-\alpha},
$$

where $r$ denotes the transmission distance in the CP. Furthermore, $A$ stands for the corresponding path gain at a reference distance of 1 meter in the CP. We set $A=1$ for simplicity.
For a user associated with its nearest CBS, the signal-tointerference ratio-plus-noise (SINR) is expressed as

$$
S I N R_{C}=\frac{P_{t}^{C} h r^{-\alpha}}{\sum_{k \in \Psi_{I}^{C}} P_{t}^{C} h_{k} r_{k}^{-\alpha}+\sigma^{2}},
$$

where $h$ and $h_{k}$ denote the channels' power gain in the desired link and in the interfering link spanning from the $k$ th interfering CBS, which follow two independent exponential distributions with unity mean, respectively. While $r$ and $r_{k}$ denote the length of the desired link and of the interfering link spanning from the $k$ th interfering CBS, respectively, and $\sigma^{2}$ denotes the additive noise. For CBSs, providing reliable CP coverage has a higher priority than offering high date transmission capacity. Hence, we analyze the coverage probability of the $\mathrm{CP}$, which is defined as the probability that the DL SINR of a user is above a predefined threshold $x_{0}$, i.e.,

$$
p^{\mathrm{cov}}\left(x_{0}, \lambda_{C}, \alpha\right)=\operatorname{Pr}\left[S I N R_{C}>x_{0}\right] .
$$

\section{Traffic Model for the User Plane}

Considering the potentially high density of TBSs, we use a path loss model to include both LoS and NLoS transmissions depending on the ranges of links. The path gain in the UP is given by

$$
\Theta^{U P}=\left\{\begin{array}{ccccc}
A^{L} r^{-\alpha^{L}} & \text { if } & \operatorname{LoS} & \text { w.p. } & P_{T}^{L}(r) \\
A^{N} r^{-\alpha^{N}} & \text { if } & \mathrm{NLoS} & \text { w.p. } & 1-P_{T}^{L}(r)
\end{array}\right.
$$

where $\alpha^{L}$ and $\alpha^{N}$ represent the path loss exponents for LoS and NLoS transmissions, respectively. The corresponding path gains at a reference distance of 1 meter in UP are given by $A_{L}$ and $A_{N}$, respectively. $P_{T}^{L}(r)$ is the LoS probability function of TBSs, which is defined as

$$
P_{T}^{L}(r)=\left\{\begin{array}{cc}
1-\frac{r}{d_{0}}, & 0<r \leq d_{0} \\
0, & r>d_{0}
\end{array},\right.
$$

where $d_{0}$ denotes the reference distance, which is given by $d_{0}=300 \mathrm{~m}$. The probability of a link with a range of $r$ being LoS or NLoS is $P_{T}^{L}(r)$ or $P_{T}^{N}(r)$, respectively, which satisfy $P_{T}^{L}(r)+P_{T}^{N}(r)=1$.

Note that because CBSs and TBSs transmit their signals in the low and high carrier frequencies, respectively, the path gain of the CP can be much larger than that of the UP. Thus, we set $A=1$ for simplicity and use the path loss exponents $\alpha, \alpha^{L}$ and $\alpha^{N}$ having different values.

If there exists a LoS path between a TBS and a typical user, then the TBS is considered as a LoS TBS. For a typical user, all the LoS TBSs form the set $\Psi_{L}$, while the other TBSs are considered to be NLoS TBSs and form the set $\Psi_{N}$. We have $\Psi_{L} \cup \Psi_{N}=\Psi_{T}$. Note that $\Psi_{L}$ and $\Psi_{N}$ are two independent non-homogeneous PPPs with densities of $\lambda_{L}(r)=P_{T}^{L}(r) \lambda_{T}$ and $\lambda_{N}(r)=P_{T}^{N}(r) \lambda_{T}$, respectively. For a typical user in the UP, if its $\Phi_{L}$ is not empty, then the user is served by the LoS TBS that offers the highest received power; otherwise, the user is served by the closest NLoS TBS. The total user traffic over an area covered by a LoS TBS and a NLoS TBS is $\nu^{L}(r)=\lambda_{b} N_{L}(r)$ and $\nu^{N}(r)=\lambda_{b} N_{N}(r)$, 
where the average numbers of users served by each LoS TBS and each NLoS TBS are $N_{L}(r)=p_{u} \lambda_{u} / \lambda_{L}(r)$ and $N_{N}(r)=p_{u} \lambda_{u} / \lambda_{N}(r)$, respectively. Each TBS has access to a total of $C_{T}$ channels, and thus a LoS TBS and a NLoS TBS can be modeled as an $M / M / C_{T} / C_{T}$ queue with an Erlang load of $a_{l}(r)=\nu^{L}(r) / \mu_{t}$ and $a_{n}(r)=\nu^{N}(r) / \mu_{t}$. The probabilities that $j \in 1, \ldots, C_{T}$ channels are being used in a LoS TBS and a NLoS TBS are $\phi_{j}^{L}(r)=\frac{\left(a_{l}(r)\right)^{j}}{j !} \phi_{0}^{L}(r)$ and $\phi_{j}^{N}(r)=\frac{\left(a_{n}(r)\right)^{j}}{j !} \phi_{0}^{N}(r)$, respectively.

Let's denote the probabilities of a neighbor LoS TBS and a neighbor NLoS TBS imposing interference on the typical user by $\rho^{L}(r)$ and $\rho^{N}(r)$ [10], respectively. We have $\rho^{L}(r)=\sum_{j=1}^{C_{T}} \frac{j}{C_{T}} \phi_{j}^{L}(r)$ and $\rho^{N}(r)=\sum_{j=1}^{C_{T}} \frac{j}{C_{T}} \phi_{j}^{N}(r)$. For the typical user, the interfering LoS TBSs and the interfering NLoS TBSs are distributed obeying two independent PPPs denoted by $\Phi_{I}^{L}$ and $\Phi_{I}^{N}$, which have densities of $\lambda_{I}^{L}(r)=$ $\rho^{L}(r) \lambda_{L}(r)$ and $\lambda_{I}^{N}(r)=\rho^{N}(r) \lambda_{N}(r)$, respectively. Every channel of a TBS has a DL transmission power $P_{t}^{T}$ per channel.

When the number of active users exceeds the number of channels available for a typical TBS, a call will be blocked. The user blocking probabilities of a LoS TBS and of a NLoS TBS are $P_{b}^{L}(r)=\phi_{C_{T}}^{L}(r)$ and $P_{b}^{N}(r)=\phi_{C_{T}}^{N}(r)$, respectively.

\section{Channel Model for the User Plane}

In the UP, a typical user associates with a TBS controlled by the maximum received signal power based user-association scheme. The SINR of a user associated with its serving LoS TBS or its serving NLoS TBS is

$$
S I N R_{U}=\frac{P_{t}^{T} A^{U} h^{U} r^{-\alpha^{U}}}{I_{T}+\sigma^{2}}
$$

where $U \in\{L, N\}, A^{U}$ is the path loss constant at a reference distance, and $\alpha^{U}$ is the path loss exponent. The channel's power gain $h^{U}$ follows an exponential distribution with unity mean; $I_{T}$ is the cumulative interference power received from all the interfering LoS TBSs and NLoS TBSs, which can be expressed as

$$
I_{T}=\sum_{j \in \Psi_{I}^{L}} P_{t}^{T} A^{L} h_{j}^{L} r_{j}^{-\alpha^{L}}+\sum_{k \in \Psi_{I}^{N}} P_{t}^{T} A^{N} h_{k}^{N} r_{k}^{-\alpha^{N}} .
$$

Because the TBSs are responsible for the high speed data transmissions, we analyze the energy efficiency [9-10] of the $\mathrm{UP}$, which is defined by $\eta_{E}=\frac{\mathrm{ASE}}{\mathrm{PCA}}$.

The ASE is quantified in terms of $\mathrm{b} / \mathrm{s} / \mathrm{Hz} / \mathrm{m}^{2}$, while PCA is the transmission power consumption per unit area (PCA) in $\mathrm{W} / \mathrm{m}^{2}$, both of which will be explained in the next section.

\section{Problem Formulation And SOlution}

In this section, we will derive the coverage of the $\mathrm{CP}$ and the energy efficiency of the UP by exploiting the properties of PPP, respectively.

\section{A. Coverage Probability of the Control Plane}

Upon substituting (3) into (4), we obtain the coverage probability of the $\mathrm{CP}$ as

$$
\begin{aligned}
& p_{C}^{\text {cov }}\left(x_{0}\right)=\int_{r>0} \mathbb{P}\left[S I N R_{C}>x_{0} \mid r\right] f_{R}(r) d r \\
& \quad=\int_{r>0} \exp \left(-\pi r^{2} \lambda_{I}^{C} Z\left(x_{0}, \alpha\right)-\frac{x_{0} \sigma^{2} r^{\alpha}}{P_{t}^{C}}\right) f_{R}(r) d r,
\end{aligned}
$$

where the probability density function (PDF) of the transmission distance between the user and its serving CBS $f_{R}(r)$ in (9) is given by $f_{R}(r)=2 \pi \lambda_{C} r e^{-\pi \lambda_{C} r^{2}}, Z\left(x_{0}, \alpha\right)=$ $x_{0}^{2 / \alpha} \int_{x_{0}-2 / \alpha}^{\infty} \frac{1}{1+u^{\alpha / 2}} d u$. (9) is exploiting the properties of the exponential distribution $h_{k} \sim \exp (1)$ [13].

\section{B. Energy Efficiency of the User Plane}

We define the event that the typical user is connected to a LoS TBS or a NLoS TBS as $X^{L}$ and $X^{N}$ with probabilities of $H^{L}$ and $H^{N}$, respectively, i.e., $H^{L}=\mathbb{P}\left[X^{L}\right]$ and $H^{N}=$ $\mathbb{P}\left[X^{N}\right]$. We have $H^{L}+H^{N}=1$. The distances between the typical user and its nearest LoS TBS as well as its nearest NLoS TBS are denoted by $D_{T}^{L}$ and $D_{T}^{N}$, respectively. Then $H^{L}$ can be expressed as

$$
\begin{aligned}
H^{L} & =\mathbb{P}\left[P_{t}^{T} A^{L}\left(D_{T}^{L}\right)^{-\alpha^{L}}>P_{t}^{T} A^{N}\left(D_{T}^{N}\right)^{-\alpha^{N}} \mid D_{T}^{L}=r\right] \\
& =2 \pi \lambda_{T} \int_{0}^{\infty} \theta^{L} r P_{T}^{L}(r) d r .
\end{aligned}
$$

Similarly, $H^{N}$ can be formulated as

$$
H^{N}=2 \pi \lambda_{T} \int_{0}^{\infty} \theta^{N} r\left(1-P_{T}^{L}(r)\right) d r
$$

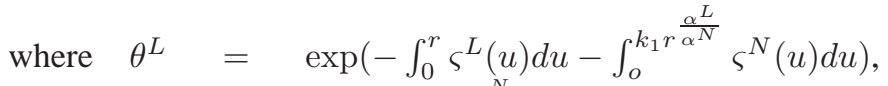
and $\theta^{N}=\exp \left(-\int_{0}^{k_{2} r^{\frac{\alpha^{N}}{\alpha^{L}}}} \varsigma^{L}(u) d u-\int_{0}^{r} \varsigma^{N}(u) d u\right)$. $k_{1}=\left(A^{L} / A^{N}\right)^{1 / \alpha^{N}}, \quad k_{2}=\left(A^{N} / A^{L}\right)^{1 / \alpha^{L}}$, $\varsigma^{L}(u)=2 \pi u \lambda_{T} P_{T}^{L}(u)$ and $\varsigma^{N}(u)=2 \pi u \lambda_{T}\left(1-P_{T}^{L}(u)\right)$.

In the UP, the user will connect to its nearest LoS TBS, namely to the one that provides a high received power instead of the nearest TBS associated with a NLoS path [14]. When the typical user is associated with a LoS TBS or a NLoS TBS, the distance between the user and its serving TBS is denoted as $R_{T}^{L}$ and $R_{T}^{N}$, respectively. Furthermore, $R_{T}^{L}>r$ and $R_{T}^{N}>r$ are equivalent to $D_{T}^{L}>r$ and $D_{T}^{N}>r$, given that the typical user is connected to a LoS TBS and a NLoS TBS, respectively. Hence the PDFs of the two distances can be expressed as

$$
f_{R_{T}^{U}}(r)=\frac{d\left(1-\left[R_{T}^{U}>r\right]\right)}{d r}=\frac{2 \pi \lambda_{T} r P_{T}^{U}(r) \theta^{U}}{H^{U}}
$$

where $U \in\{L, N\}$.

According to (9), given a predefined threshold $x_{0}$, the coverage probability of a user served by a LoS TBS is given by

$$
p_{L}^{c o v}\left(x_{0}\right)=\int_{r>0} \exp \left(-\frac{x_{0} \sigma^{2} r^{\alpha^{L}}}{P_{t}^{T} A^{L}}\right) \mathcal{L}_{I_{T}}\left(\frac{x_{0} r^{\alpha^{L}}}{P_{t}^{T} A^{L}}\right) f_{R_{T}^{L}}(r) d r,
$$


where $f_{R_{T}^{L}}(r)$ is provided in (12). According to the properties of PPPs, the Laplace transform of $I_{T}$ in (8) is obtained as

$$
\begin{gathered}
\mathcal{L}_{I_{T}}(s)=E_{h_{k}}\left[\exp \left(-s \sum_{k \in \Psi_{I}^{L}} P_{t}^{T} A^{L} h_{k}^{L} r_{k}^{-\alpha^{L}}\right)\right] \\
\times E_{h_{j}}\left[\exp \left(-s \sum_{j \in \Psi_{I}^{N}} P_{t}^{T} A^{N} h_{j}^{N} r_{j}^{-\alpha^{N}}\right)\right] .
\end{gathered}
$$

Substituting $s=\frac{x_{0} r^{\alpha^{L}}}{P_{t}^{T} A^{L}}$ into (14), we arrive at

$$
\begin{aligned}
& \mathcal{L}_{I_{T}}\left(\frac{x_{0} r^{\alpha^{L}}}{P_{t}^{T} A^{L}}\right)=\exp \left(-\pi r^{2} \lambda_{I}^{L}(r) Z\left(x_{0}, \alpha^{L}\right)\right) \\
& \times \exp \left(-\pi r^{2} \lambda_{I}^{N}(r) Z\left(\frac{x_{0} A^{N}}{A^{L}}, \alpha^{N}\right)\right) .
\end{aligned}
$$

Similarly, we obtain the expression of the coverage probability $p_{N}^{c o v}\left(x_{0}\right)$, which is the same as $p_{L}^{c o v}\left(x_{0}\right)$, except that $\lambda_{L}, A^{L}$ and $\alpha^{L}$ are replaced by $\lambda_{N}, A^{N}$ and $\alpha^{N}$.

Upon substituting $s=\frac{x_{0} r^{\alpha^{N}}}{P_{t}^{T} A^{N}}$ into (14), we have

$$
\begin{gathered}
\mathcal{L}_{I_{T}}\left(\frac{x_{0} r^{\alpha^{N}}}{P_{t}^{T} A^{N}}\right)=\exp \left(-\pi r^{2} \lambda_{I}^{L}(r) Z\left(\frac{x_{0} A^{L}}{A^{N}}, \alpha^{L}\right)\right) \\
\times \exp \left(-\pi r^{2} \lambda_{I}^{N}(r) Z\left(x_{0}, \alpha^{N}\right)\right) .
\end{gathered}
$$

In (15) and (16), $Z\left(x_{0}, \alpha^{L}\right), \quad Z\left(\frac{x_{0} A^{N}}{A^{L}}, \alpha^{N}\right)$, $Z\left(\frac{x_{0} A^{L}}{A^{N}}, \alpha^{L}\right)$ and $Z\left(x_{0}, \alpha^{N}\right)$ follow the expression of $Z\left(x_{0}, \alpha\right)$, as defined for (9).

We denote the area spectral efficiencies provided by LoS TBSs and NLoS TBSs, respectively, as $\eta^{L}$ and $\eta^{N}$, which are expressed as

$$
\eta^{U}=\mathbb{E}\left[\log _{2}\left(1+S I N R_{U}\right)\right]=\frac{1}{\operatorname{In2}} \int_{0}^{\infty} \frac{p_{U}^{c o v}\left(x_{0}\right)}{x_{0}+1} d x_{0} .
$$

We denote the expectations of user blocking probabilities by $P_{b}^{L}$ and $P_{b}^{N}$, which represent the average probability of a typical user's call being blocked by a LoS TBS or a NLoS TBS, respectively. Then $P_{b}^{L}$ and $P_{b}^{N}$ can be calculated as

$$
\begin{aligned}
& P_{b}^{L}=\int_{0}^{\infty} P_{b}^{L}(r) f_{R_{T}^{L}}(r) d r, \\
& P_{b}^{N}=\int_{0}^{\infty} P_{b}^{N}(r) f_{R_{T}^{N}}(r) d r .
\end{aligned}
$$

For a given TBS intensity $\lambda_{T}$, the ASE in the UP is expressed as follows

$$
\mathrm{ASE}=p_{u} \lambda_{u}\left(1-P_{b}^{L}\right) H^{L} \eta^{L}+p_{u} \lambda_{u}\left(1-P_{b}^{N}\right) H^{N} \eta^{N} .
$$

The PCA in the UP can be expressed as

$$
\mathrm{PCA}=\int_{0}^{\infty}\left(\lambda_{L}(r) \varpi^{L}(r) f_{R_{T}^{L}}(r)+\lambda_{N}(r) \varpi^{N}(r) f_{R_{T}^{N}}(r)\right) d r
$$

where $\varpi^{L}(r)$ and $\varpi^{N}(r)$ denote the power consumptions of a LoS TBS and a NLoS TBS, respectively, which are given by

$$
\varpi^{U}(r)=\sum_{j=1}^{C^{T}} j P_{t}^{T} \phi_{j}^{U}(r)+P_{O M} \phi_{0}^{U}(r),
$$

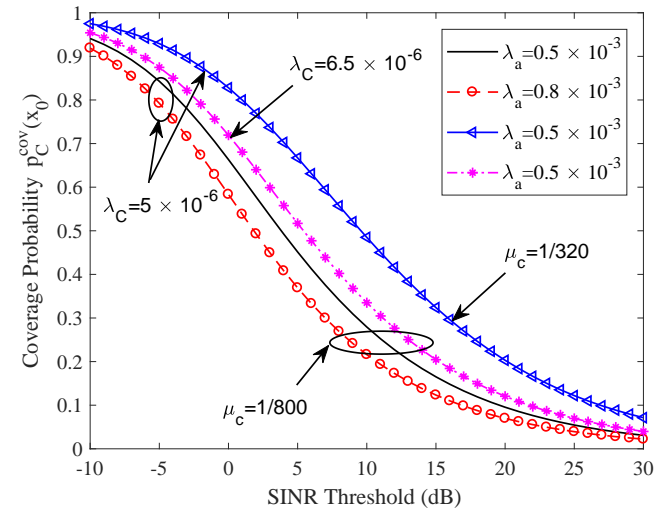

Fig. 1. The coverage probability of the CP vs. the SINR threshold $\lambda_{T}$.

where $U \in\{L, N\}$ and $P_{O M}$ is the power assigned to cooling and other mains-operated functions. If no user requests any service within a TBS's coverage, no channel will be occupied. Therefore, the TBS remains sleeping state consuming a power of $P_{O M}$.

Combining (20) and (21), we obtain the energy efficiency of the UP as

$\eta_{E}=\frac{p_{u} \lambda_{u}\left(1-P_{b}^{L}\right) H^{L} \eta^{L}+p_{u} \lambda_{u}\left(1-P_{b}^{N}\right) H^{N} \eta^{N}}{\int_{0}^{\infty}\left[\lambda_{L}(r) \varpi^{L}(r) f_{R_{T}^{L}}(r)+\lambda_{N}(r) \varpi^{N}(r) f_{R_{T}^{N}}(r) d r\right] d r}$.

\section{NUMERICAL RESULTS AND DisCUSSIONS}

In this section, we present our numerical results to show the performance of cellular RANs relying on CUPS in accordance with our theoretical analysis. According to [16] - [18], we use the parameters $P_{t}^{C}=1 \mathrm{~W}, P_{t 1}^{T}=0.4 \mathrm{~W}, P_{O M}=0.25 \mathrm{~W}$, $\sigma^{2}=-90 \mathrm{dBm}, A^{L}=10^{-4.11}, A^{N}=10^{-3.01}, \alpha=4, \alpha^{L}=$ $2.5, \alpha^{N L}=3.5, C_{C}=50, C_{T}=5, p_{u}=0.3, d_{0}=300$, $\lambda_{a} \in\left\{0.5 \times 10^{-3}, 0.8 \times 10^{-3}\right\}, \lambda_{b} \in\left\{4 \times 10^{-3}, 5 \times 10^{-3}\right\}$, $1 / \mu_{c} \in\{800,320\}$ (sec), and $1 / \mu_{t} \in\{80,64\}$ (sec), unless specified otherwise.

Fig. 1 shows the coverage probability of the CP versus the SINR threshold $x_{0}$, for various $\lambda_{a}, \mu_{c}$ and $\lambda_{C}$ values. Note that the coverage probability increases with increasing the service rate $\mu_{c}$ and $\lambda_{C}$, or with reducing either the access rate $\lambda_{a}$ or the SINR threshold $x_{0}$. The reduction of $\lambda_{a}, \lambda_{C}$ and $\mu_{c}$ leads to an increase in $\lambda_{I}^{C}$, causing a decrease in the coverage probability. Therefore, when the arrival rate of user requests is high, a higher coverage probability can be achieved by increasing the CBSs' deployment density or by increasing each CBS's service rate.

As illustrated in Fig. 2, when the TBSs are sparsely deployed (from $0.5 \times 10^{-5}$ to $1.2 \times 10^{-5} \mathrm{BSs} / \mathrm{m}^{2}$ ) and thus the interference is limited, the ASE of the UP increases quickly due to the reduction of the transmission distance and path loss. Upon the further increasing the density from $1.2 \times 10^{-5}$ to $2 \times 10^{-5} \mathrm{BSs} / \mathrm{m}^{2}$, the ASE shows a slight downward trends. Because the average radius of a TBS coverage area ranges from about $126 \mathrm{~m}$ to about $162 \mathrm{~m}$ in this density region, a user is connected to a LoS TBS with a high probability. The path loss model of interference is changed from NLoS 


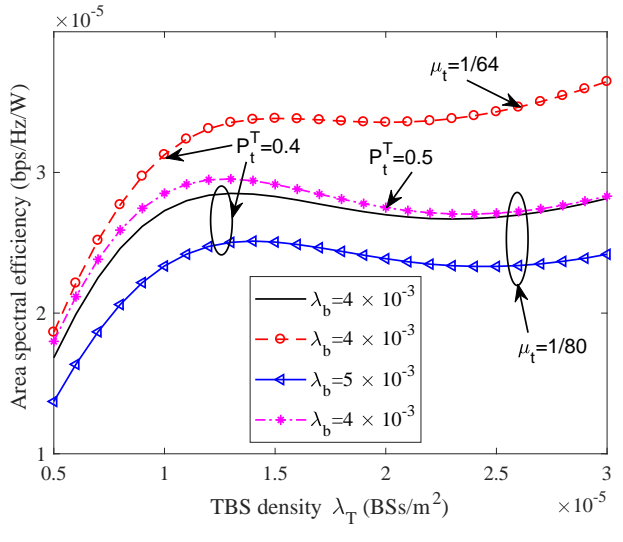

Fig. 2. The ASE of the user plane vs. the TBSs' density $\lambda_{T}$.

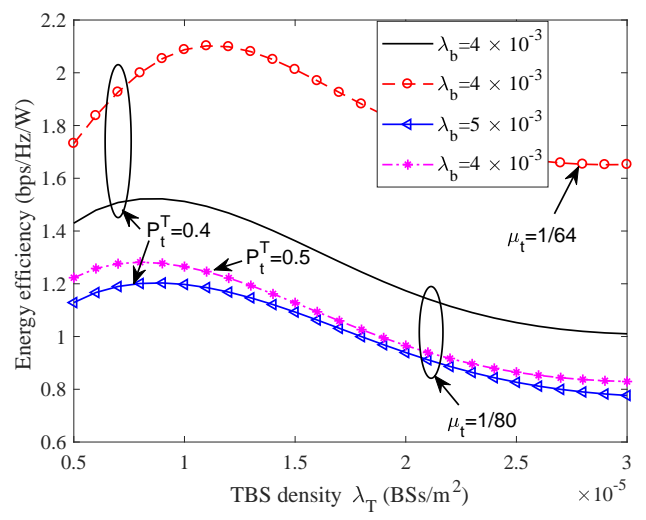

Fig. 3. The energy efficiency of the UP vs. the TBSs' density $\lambda_{T}$.

to LoS, thereby increasing the interference imposed on the user. When the TBSs are deployed more densely, the coverage probability tends to a constant upon increasing $\lambda_{T}$ (from $2 \times 10^{-5}$ to $3 \times 10^{-5} \mathrm{BSs} / \mathrm{m}^{2}$ ), because increasing the LoS TBS power is counter-balanced by the increased interference power. Therefore, the ASE shows an increasing trend. Similar trends can be found in [19] and [20].

Fig. 3 illustrates the energy efficiency of the UP versus the TBS density $\lambda_{T}$ for various $\lambda_{b}, \mu_{t}$ and $P_{t}^{T}$ values. Intuitively, there exits a TBS density $\lambda_{T}^{*}$ that maximizes the energy efficiency. More specifically, if $\lambda_{T}<\lambda_{T}^{*}$, the energy efficiency increases with respect to $\lambda_{T}$. By contrast, if $\lambda_{T}>\lambda_{T}^{*}$, the energy efficiency decreases slowly with $\lambda_{T}$. Observe from Fig.1(b) and Fig.1(c), that the UP cannot simultaneously attain the highest ASE and the highest energy efficiency at the same density $\lambda_{T}$. The maximum energy efficiency of the UP can be achieved by optimizing the TBS density. When the arrival rate $\lambda_{b}$ is high, it is necessary to appropriately reduce $P_{t}^{T}$ or increase $\mu_{t}$ in order to increase the energy efficiency.

\section{CONCLUSIONS}

In this paper, we have analyzed the performance of cellular RANs relying on CUPS, where the CP and the UP have different dynamic traffic load requirements and channel models. By relying on a sophisticated combination of stochastic geometry and queuing theory, we derive the coverage probability of the
$\mathrm{CP}$ and the energy efficiency of the UP. Interestingly, base on numerical results, we have found that increasing the CBSs' deployment density or service rates increases the coverage probability of the $\mathrm{CP}$, but there is a performance-cost tradeoff. Although the ASE and the energy efficiency of the UP cannot be simultaneously maximized by optimizing the density of TBS, the maximum energy efficiency of the UP can be achieved by optimizing the density of TBSs, while achieving a high ASE.

\section{REFERENCES}

[1] S. Zhou, T. Zhao, Z.-S Niu and S. Zhou, "Software-Defined HyperCellular Architecture for Green and Elastic Wireless Access," IEEE Commun. Mag., vol. 54, no. 1, pp. 12 - 19, Jan. 2016.

[2] A. Mohamed, et al., "Control-data separation architecture for cellular radio access networks: a survey and outlook," IEEE Communications Surveys \& Tutorials, vol. 18, no.1, pp.446-465, 2016.

[3] B. A. A. Nunes et al, "A Survey of Software-Defined Networking: Past, Present, and Future of Programmable Networks," IEEE Commun. Surv. Tut., vol. 16, no. 3, pp.1617-1634, Feb. 2014.

[4] J. Wang, J. Xu, Y. Yang and Haidong Xu, "GPP based open cellular network towards 5G," China Communications, vol. 14, no. 6, pp. 189198, Jun. 2017.

[5] J. Zhang, et al, "Energy Efficiency Analysis of Heterogeneous Cacheenabled 5G Hyper Cellular Networks," IEEE Globecom., 10.1109/GLOCOM.2016.7841790, Feb. 2016.

[6] Q. Li, W.-N Shi, X. Ge and Z. Niu, "Cooperative Edge Caching in Software-Defined Hyper-Cellular Networks," IEEE Trans. Wireless Commun., vol. 14, no. 10, pp. 5440 - 5453, Oct. 2015.

[7] S. Zhang, J. Gong, S. Zhou and Z. Niu, "How Many Small Cells Can be Turned Off via Vertical Offloading Under a Separation ," IEEE Trans. Wireless Commun., vol. 14, no. 10, pp. 5440 - 5453, Oct. 2015.

[8] B. Yang, G. Mao, M. Ding, X. Ge and X. Tao, "Dense Small Cell Networks: From Noise-Limited to Dense Interference-Limited," IEEE Trans. Veh. Tech., vol. 67, no. 5, pp. 4262-4277, May 2018.

[9] H. Zhao, L. Zhao, K. Liang and C. Pan, "Radio access network slicing based on C/U plane separation," China Communications, vol. 14, no. 12, pp. 134-141, Dec. 2017.

[10] J. B. Seo and S. Y. Kim, "Profit Versus Energy Efficiency Maximization in Regular Topology Cellular Networks," IEEE Commun. Lett., vol. 20, no. 8, pp. 1643-1646, Aug. 2016.

[11] G. Zhao, S. Chen, L. Zhao and L. Hanzo, "Energy-Spectral-Efficiency Analysis and Optimization of Heterogeneous Cellular Networks: A LargeScale User-Behavior Perspective," IEEE Trans. Veh. Tech., vol. 67, no. 5, pp. 4098-4112, May 2018.

[12] L. Wei, R. Q. Hu, Y. Qian and G. Wu, "Energy Efficiency and Spectrum Efficiency of Multihop Device-to-Device Communications Underlaying Cellular Networks," IEEE Trans. Veh. Tech., vol. 65, no. 1, pp. 367-380, Jan. 2016.

[13] J. G. Andrews, F. Baccelli, and R. K. Ganti, "A Tractable Approach to Coverage and Rate in Cellular Networks," IEEE Trans. Commun., vol. 59, no. 11, pp. 3122-3134, Nov. 2011.

[14] Q. Zhang, H. H. Yang, T. Q. Quek and J. Lee, "Heterogeneous Cellular Networks with LoS and NLoS Transmissions - The Role of Massive MIMO and Small Cells," IEEE Trans. Wireless Commun, vol. 16, no. 12, pp. 7996 - 8010, Sep. 2017.

[15] H. Ibrahim, H. Elsawy , U. T. Nguyen and M. S. Alouini, "MobilityAware Modeling and Analysis of Dense Cellular Networks With $C$ Plane/ U -Plane Split Architecture," IEEE Trans. Commun., vol. 64, no. 11, pp. 4879-4894, Nov. 2016.

[16] Spatial Channel Model AHG (Combined ad-hoc from 3GPP \& 3GPP2), "Spatial Channel Model Text Description," Apr. 2003.

[17] 3GPP, "TR 36.828 (V11.0.0): Further enhancements to LTE Time Division Duplex (TDD) for Downlink-Uplink (DLUL) interference management and traffic adaptation," Jun. 2012.

[18] J. B. Seo, et al, "Pricing in Small Cell Deployment," IEEE Commun. Lett., vol. 20, no. 8, pp. 1615-1618, Aug. 2016.

[19] P. Wang, et al, "Performance Impact of LoS and NLoS Transmissions in Dense Cellular Networks," IEEE Trans. Veh. Tech., vol. 15, no. 3, pp. 2365 - 2380, Mar. 2016.

[20] A. H. Jafari, D. Lopez-Perez, M. Ding and I. Zhang, "Performance Analysis of Dense Small Cell Networks with Practical Antenna Heights under Rician Fading," IEEE Access, vol. 6, pp. 9960 - 9974, Oct. 2017. 